\title{
Electron Spin Resonance Studies of Erythrocytes from Patients with Duchenne Muscular Dystrophy
}

\author{
Bunzo Sato, Koichi Nishikida, Leo T. Samuels, and Frank H. Tyler, Metabolic \\ Division, Department of Internal Medicine, Department of Biochemistry, and \\ Department of Chemistry, University of Utah, Salt Lake City, Utah 84132
}

A B S TRACT The membrane organization of the erythrocytes from patients with Duchenne muscular dystrophy was studied by means of electron spin resonance. The fluidity of the membrane near the polar region of Duchenne muscular dystrophy erythrocytes was similar to that of normal erythrocytes. The membrane environment in the nonpolar region, however, was quite different from that of normal erythrocytes, judged by the spectra with 2-(14carboxytetradecyl) - 2 - ethyl - 4,4 - dimethyl - 3 - oxazolidinyloxyl as probe. The temperature dependence of the ratio of the line height of central field to that at the low field showed two inflection points in normal erythrocytes at $\mathrm{pH} 7.4\left(13.5^{\circ}-16.5^{\circ}\right.$ and $37.5^{\circ}-40.5^{\circ} \mathrm{C}$, respectively) but the inflection point in the lower temperature range was not detected in Duchenne muscular dystrophy erythrocytes. When $\mathrm{pH}$ was varied, an abrupt decrease in the ratio was observed at $\mathrm{pH}$ 5.9-5.6 in normal erythrocytes whereas there was a gradual decrease over the range of $\mathrm{pH}$ from 6.6 to 5.0 in Duchenne muscular dystrophy erythrocytes.

The rate of reduction of the radical 2-(3-carboxypropyl)-4,4-dimethyl-2-tridecyl-3-oxazolidinyloxyl by ascorbate in normal erythrocytes was faster than that in Duchenne muscular dystrophy erythrocytes. Treatment of both erythrocytes with phloretin markedly reduced the rate of reduction by ascorbate and eliminated the difference in the two types of erythrocyte. These results indicate that in Duchenne muscular dystrophy the erythrocyte membrane is involved as well as the muscle cell.

\section{INTRODUCTION}

Duchenne muscular dystrophy (DMD) ${ }^{1}$ is a progressive disorder inherited as an $\mathrm{X}$-linked recessive trait. It

Received for publication 27 May 1977 and in revised form 7 October 1977.

${ }^{1}$ Abbreviations used in this paper: BSA, bovine serum albumin; Buffer I, 0.15 M NaCl-0.01 M Tris, pH 7.4; DMD, is associated with proximal muscle weakness and atrophy, cardiac involvement, and a high rate of varying degrees of mental retardation (1). Elevated levels of muscle enzyme activities in the serum and their depletion in the muscle tissue have implied an abnormality in the plasma membrane as the probable site of the genetic defect, although the primary inherited metabolic defect is unknown. The elucidation of the primary biochemical defect with muscle specimens obtained from patients by biopsy is made extremely difficult by the presence of atrophy, fibrous tissue, the change in innervation that may cause multiple secondary biochemical changes, and by the small yield.

Recently, marked surface deformation of erythrocytes from DMD patients was observed by Matheson and Howland (2). This observation was consistent with previous results indicating possible abnormalities in cation transport (3) and lipid patterns (4) in erythrocyte membranes of DMD patients. Cell elastimetry also showed abnormal deformity to the erythrocytes of patients with DMD (5). This evidence indicated that the membrane abnormality also involved erythrocytes. The biochemical studies by Roses et al. (6) on phosphorylation in membrane proteins of erythrocytes from DMD patients also supports this general conclusion.

The electron spin resonance (ESR) technique has been well established as a valuable tool in the study of both biological membranes and model membranes since its introduction by McConnell and his co-workers in the middle 1960's. A multitude of publications show it has been applied with success to the erythrocyte membrane. The interesting studies by Butterfield et al. (7-9) implied differences in the spin label signals of erythrocytes from normal subjects and patients with

Duchenne muscular dystrophy; ESR, electron spin resonance; G, gauss; I(12.3), 2-(3-carboxypropyl)-4,4-dimethyl-2-tridecyl3-oxazolidinyloxyl; I(5.10), 2-(10-carboxydecyl)-2-hexyl-4,4dimethyl-3-oxazolidinyloxyl; I(1.14), 2-(14-carboxytetradecyl)2-ethyl-4,4-dimethyl-3-oxazol-idinyloxyl. 
myotonic muscular dystrophy. From these reports we decided to employ the ESR technique to examine the membrane organization of the erythrocytes from DMD patients compared with those of normal erythrocytes.

\section{METHODS}

13 Patients ranging from 6 to $14 \mathrm{yr}$ of age were studied in the Medical Center, Muscle Clinic, University of Utah. The diagnosis of DMD was established by clinical history and examination, serum enzymes, electromyography, and muscle biopsy. As controls, normal males matched for age were used. Heparinized blood from patients and paired controls was obtained at almost the same time by venipuncture and plasma was removed by centrifugation. The cells were then washed three times in $0.15 \mathrm{M} \mathrm{NaCl-0.01} \mathrm{M} \mathrm{Tris,} \mathrm{pH} 7.4$ (Buffer I). Heparin did not affect ESR spectra when compared with those erythrocytes prepared without any anticoagulant by immediate centrifugation after blood was drawn. The washed erythrocytes from both patients and controls were stored at $4^{\circ} \mathrm{C}$ for 10-12 h in Buffer I and used for measurements of ESR spectra within $24 \mathrm{~h}$ after venipuncture.

To label the erythrocyte membrane with a spin labeled stearic acid derivative, the bovine serum albumin exchange method was used (10). Bovine serum albumin (BSA) solution (5\% in Buffer I) was added to a Corex tube (Corning Glass Works, Science Products Div., Corning, N. Y.), the bottom of which had a thin film of spin labeled stearic acid derivative ( $1 \mathrm{mg} / \mathrm{ml}$ of BSA solution), followed by incubation at room temperature $\left(22^{\circ} \mathrm{C}\right)$ for $12 \mathrm{~h}$ in the dark to form a BSA-fatty acid complex. Packed erythrocytes were then incubated with an equal volume of this BSA-fatty acid complex at room temperature for $2 \mathrm{~h}$. The cells were again separated by centrifugation at $1,500 \mathrm{~g}$ for $10 \mathrm{~min}$ at $4^{\circ} \mathrm{C}$ and washed three times with 10 vol of Buffer I. When studying effects of $\mathrm{pH}$ on membrane fluidity, the packed erythrocytes with the spin labeled fatty acid derivatives incorporated were washed twice more with $10 \mathrm{vol}$ of the indicated buffers (the details are given in the figure legends). During these procedures, the amount of hemolysis was $<2 \%$ except when low $\mathrm{pH}$ was used, which caused increasing amounts of hemolysis; however, this did not exceed $5 \%$.

To measure the reduction of the nitroxide radicals incorporated into the erythrocytes, $10 \mu \mathrm{l}$ of $30 \mathrm{mM}$ sodium ascorbate solution (in Buffer I, $\mathrm{pH} 7.4$, freshly prepared) was added to 50 $\mu l$ of the packed erythrocytes in Buffer I. After rapid mixing, an aliquot was used to record the ESR spectra repeatedly at l-min intervals.

The effect of phloretin on the reduction of the radicals by ascorbate was also studied. The phloretin was dissolved in ethanol and then diluted with the appropriate volume of Buffer I. This solution was added to the packed erythrocytes in Buffer I in which the spin labeled stearic acid had been incorporated under the same conditions as described above. The final concentrations of phloretin and ethanol were $0.5 \mathrm{mM}$ and $0.5 \%$ ( $\mathrm{vol} / \mathrm{vol})$, respectively. The concentrations of both reagents did not interfere with the ESR spectra. The erythrocytes were incubated in the phloretin solution for $\cong 90 \mathrm{~min}$ at room temperature, followed by a study of the reduction kinetics of the radicals by ascorbate as described above.

The ESR spectra were obtained using a Varian $4502 \mathrm{X}$ band spectrometer (Varian Associates, Palo Alto, Calif.) equipped with a $100-\mathrm{kH}_{\mathrm{z}}$ field modulation and control unit. The temperature was controlled by a Varian 4540 temperature control unit (Varian Associates) and measured by a copperconstantan thermocouple. Temperatures were accurate to within $\pm 1^{\circ} \mathrm{C}$. The usual spectrometer settings were modula- tion amplitude, 1 gauss (G); scan range, $100 \mathrm{G}$; filter time constant, $0.3 \mathrm{~s}$; scan speed, $5 \mathrm{~min}$. When the reduction kinetics of the radicals by ascorbate were studied, the spectra of the central field (Fig. 1) were scanned every minute with $10 \mathrm{G} / \mathrm{min}$ as a scanning speed. The sample was sealed in a capillary which was immersed in a cylindrical sample holder and permitted to stand for at least $3 \mathrm{~min}$ at each desired temperature. The amount of hemoglobin released was determined by measuring the absorbance at a wavelength of $543 \mathrm{~nm}$. The fraction of cells
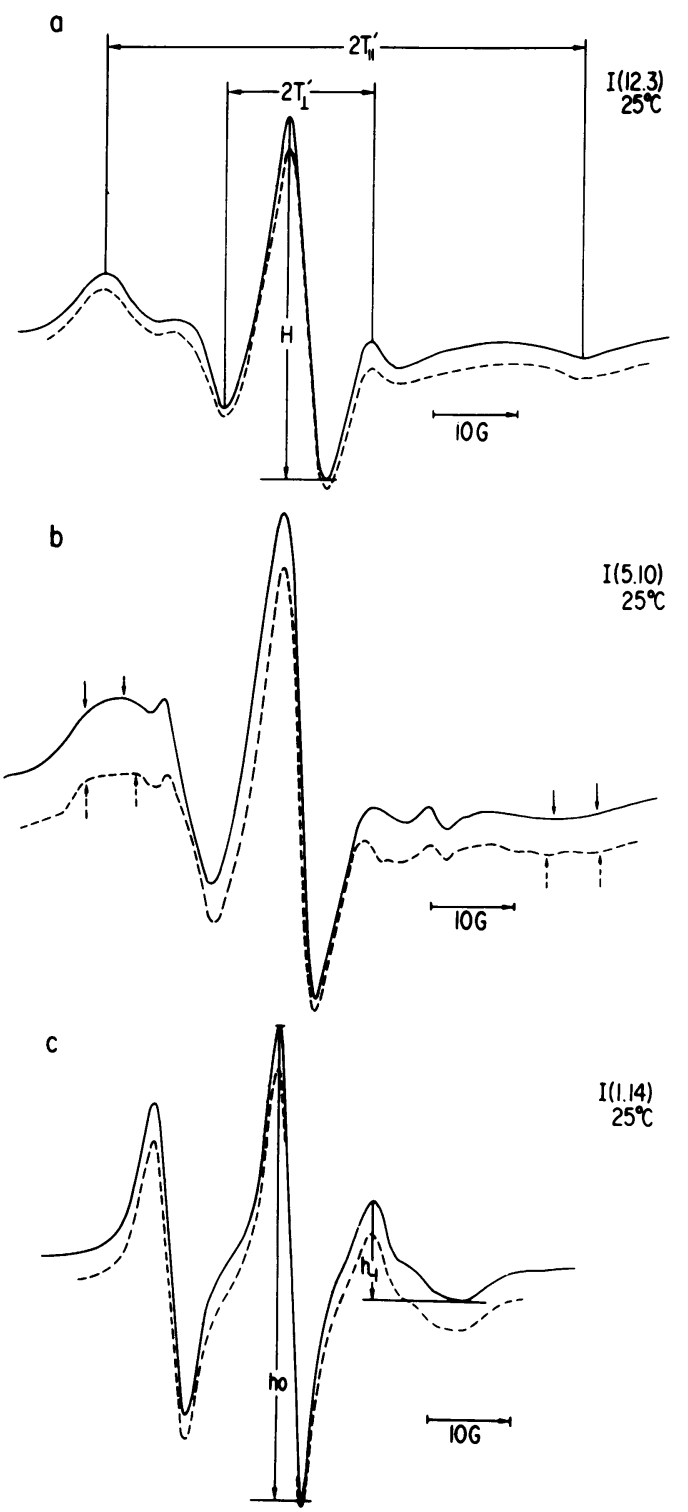

FIgURE 1 ESR spectra at $25^{\circ} \mathrm{C}$ for fatty acid spin labels incorporated into erythrocytes. ESR spectra of fatty acid probes incorporated into normal (-) or DMD patients' $(--)$ erythrocytes were recorded at $25^{\circ} \mathrm{C}$. (a) $\mathrm{I}(12.3),(b)$ $\mathrm{I}(10.5),(c) \mathrm{I}(1.14)$. The parameters used for interpreting each spectrum are as shown on the spectrum obtained by incorporating the probe into normal erythrocytes. The arrows on the spectra of $\mathrm{I}(5.10)$ show the distortion due to two peaks both at the low and at the high field. (See text). 
lysed was calculated from the hemoglobin released by complete lysis of the same volume of packed cells with $0.01 \mathrm{M}$ Tris, pH 7.4.

Spin labeled stearic acid derivatives were purchased from Syva Associated, Palo Alto, Calif. The general molecular formula was as follows:

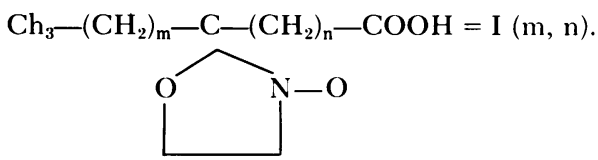

BSA, essentially fatty acid-free, was obtained from Sigma Chemical Co., St. Louis, Mo., and phloretin was purchased from K \& K Laboratories Inc., Plainview, N. Y. The other chemicals used were of an analytical grade.

\section{RESULTS}

ESR spectra at $25^{\circ} \mathrm{C}$ of the three probes incorporated into either normal or DMD erythrocytes are shown in Fig. 1. Spectra of 2-(3-carboxypropyl-4,4-dimethyl2-tridecyl-3-oxazolidinyloxyl [I(12.3)] were typical of rapid anisotropic motion over a wide range of temperatures. The separation between the outermost extremes corresponds to $2 \mathrm{~T}^{\prime} \|$ (Fig. 1) which has been established as a good parameter of fluidity (11). The order parameter $S$ was also used as a parameter of fluidity, calculated by the following equation (12):

$$
\mathrm{S}=\frac{\mathrm{T}^{\prime} \|-\mathrm{T}^{\prime}}{\mathrm{T}_{z z}-\mathrm{T}_{x x}} \cdot \frac{a_{n}}{a^{\prime}{ }_{n}},
$$

where $\mathrm{T}_{z z}(30.8 \mathrm{G})$ and $\mathrm{T}_{x x}(5.8 \mathrm{G})$ are the maximum and the minimum possible splittings of the outer and inner pair of lines, respectively. To compensate for polarity differences in different environments, it was normalized by the factor $a_{n} / a_{n}{ }^{\prime}, a$ being the isotropic coupling constant for the label appropriate to the system being studied (12). The accurate determination of $T_{\perp}{ }^{\prime}$ at low temperatures, however, was difficult.

The ESR spectra of 2-(10-carboxydecyl)-2-hexyl-4, 4-dimethyl-3-oxazolidinyloxyl [I(5.10)] for normal and DMD erythrocytes were similar to those of $\mathrm{I}(12.3)$ at temperatures below $15^{\circ} \mathrm{C}$. When the temperature was higher than $15^{\circ} \mathrm{C}$, however, the extreme peaks at the highest and lowest magnetic fields became broad and resulted in a split into two peaks, especially at the highest fields (Fig. 1). These results suggest heterogeneous distribution of the radicals of this probe, each of which showed anisotropic motion, but with different degrees of freedom.

The ESR spectra of 2-(14-carboxytetradecyl)-2-ethyl4,4-dimethyl-3-oxazolidinyloxyl [I(1.14)] at high temperatures showed an isotropic triplet hyperfine structure (13) with a distortion due to incomplete averaging of anisotropic hyperfine interaction. As temperature decreased, the asymmetry of the peak at the highest field became more prominent (Fig. 2).

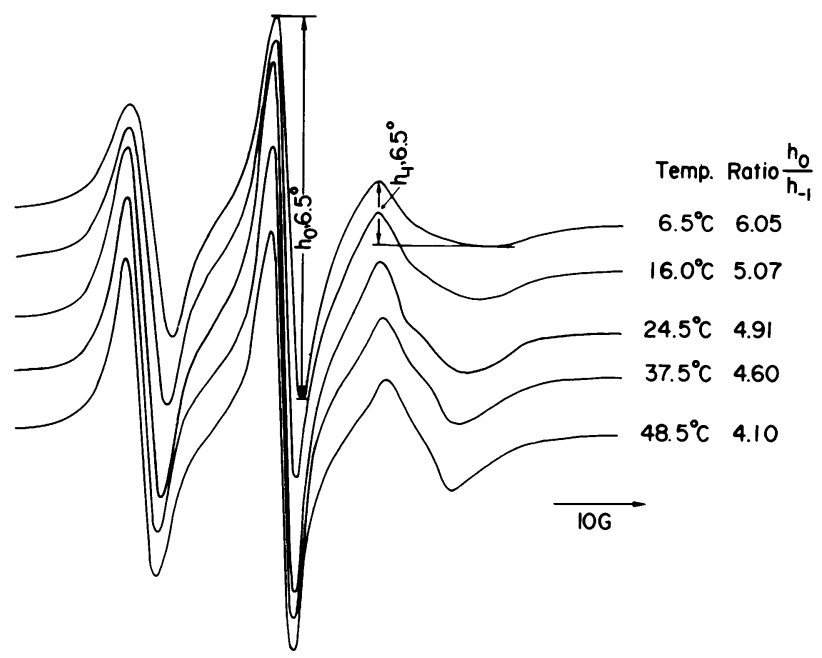

Figure 2 Temperature dependence of the spectra of $\mathrm{I}(1.14)$. The spectra were obtained by incorporating $\mathrm{I}(1.14)$ into normal erythrocytes and recording at various temperatures.

Thus, spectra such as are shown in Figs. 1 and 2 are difficult to interpret quantitatively. The rigid formula of Stone et al. (14) is difficult to apply to our system due to a molecular tumbling speed much slower than $10^{-9} \mathrm{~s}$, the lower limit for the equation's validity, and also the presence of some distortion by anisotrophy. However, since, as seen in Fig. 2, $\mathrm{h}_{-1}$, the peak height of the high field line, increases more rapidly with temperature than $h_{0}$, the peak height of the midfield line, the ratio of peak heights, $\mathrm{h}_{0} / \mathrm{h}_{-1}$, may be used as an empirical relative measurement of fluidity. This parameter shows sharp temperature dependence (see below). The spectra of I(1.14) incorporated into erythrocytes have been treated as anisotropy in many papers and $2 \mathrm{~T}^{\prime} \mathrm{p}$ has been calculated from the distance between the peak at low field and the hollow at high field. As indicated by Ito and Ohnishi (15), however, $2 \mathrm{~T}^{\prime} \|$ values cannot be measured accurately from the spectra because the low field peak is not resolved.

Several parameters obtained from the spectra of $\mathrm{I}(12.3)$ and $\mathrm{I}(1.14)$ at $5^{\circ}$ and $30^{\circ} \mathrm{C}$ are summarized in Table I. No parameters of the spectra of $\mathrm{I}(12.3)$ showed a significant difference between normal and DMD erythrocytes. On the other hand, $h_{0} / h_{-1}$ of the spectra of $\mathrm{I}(1.14)$ in DMD erythrocytes differed significantly from that in normal erythrocytes, both at $5^{\circ} \mathrm{C}$ and at $30^{\circ} \mathrm{C}$.

The ESR spectra of $\mathrm{I}(12.3)$ and $\mathrm{I}(1.14)$ of four patients and four paired controls were measured at intervals of $2.5^{\circ} \mathrm{C}$ from $2^{\circ}$ to $52^{\circ} \mathrm{C} .2 \mathrm{~T}^{\prime} \|$ of the spectra of $\mathrm{I}(12.3)$ were plotted against temperature. The values for patients and controls were very close at all temperatures with no significant difference between them at any point.

The logarithms of the empirical parameter, $h_{0} / h_{-1}$ of the spectra of $\mathrm{I}(1.14)$ were plotted against the reciprocal 
TABLE I

The Various Parameters of the Spectra of I(12.3) or I(1.14) in Normal or DMD Patients' Erythrocytes at $5^{\circ}$ or $30^{\circ} \mathrm{C}$

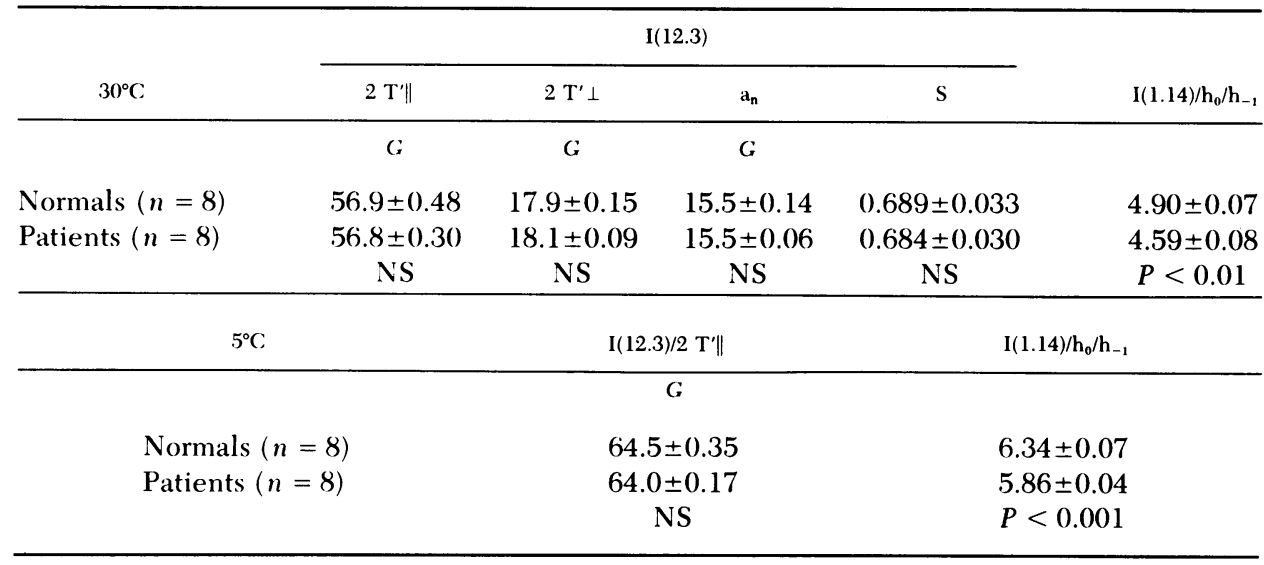

The various parameters were determined from the spectra of $\mathrm{I}(12.3)$ or $\mathrm{I}(1.14)$ at $30^{\circ}$ or at $5^{\circ} \mathrm{C}$. The statistic analysis was performed by the Student's $t$ test. The values given here are means $\pm \mathrm{SE}$.

of the absolute temperature (Fig. 3). This parameter showed definite temperature dependence. There are clearly two inflection points for normal erythrocytes, $13.5^{\circ}-16.5^{\circ} \mathrm{C}$ and $37^{\circ}-40.5^{\circ} \mathrm{C}$, respectively. The data obtained with DMD erythrocytes are in marked contrast to those with normal erythrocytes: there is no break observed at the lower temperature $\left(13.5^{\circ}-16.5^{\circ} \mathrm{C}\right)$. The erythrocytes of two patients also lacked the high temperature inflection point. The other two patients' erythrocytes showed only one inflection point at a higher temperature $\left(45.5^{\circ} \mathrm{C}\right)$ than the high temperature inflection point observed in normal erythrocytes $\left(37^{\circ}-40.5^{\circ} \mathrm{C}\right)$. These results indicate that the patients' erythrocytes permit quite different spin motion of $\mathrm{I}(1.14)$ from that in normal erythrocytes.

The temperature dependence of the spectra obtained by incorporating I(5.10) into both erythrocytes was also analyzed. As already mentioned, the peaks became broad at temperatures $>15^{\circ} \mathrm{C}$. The minimum values of 2 $\mathrm{T}^{\prime} \|$ were used as a parameter of fluidity, because accurate measurement of $2 \mathrm{~T}^{\prime} \|$ representing a relatively rigid domain was rather difficult, probably due to location of higher amounts of probe in the fluid domain. The patients' erythrocytes seemed to give more freedom to the radicals of $\mathrm{I}(5.10)$ than normal erythrocytes above $15^{\circ} \mathrm{C}$ but because only two dystrophy cases were studied the difference was uncertain.

Effects of $\mathrm{pH}$ on the spectra of $\mathrm{I}(12.3)$ and $\mathrm{I}(1.14)$ were studied. Lowering the $\mathrm{pH}$ from 7.4 to 5.0 induced only minimal changes in the spectra of $\mathrm{I}(12.3)$ both in normal and DMD erythrocytes (Fig. $4 a$ ), whereas the same treatment of both types of erythrocyte labeled with $\mathrm{I}(1.14)$ resulted in marked changes in the spectra at high field strengths (Fig. $4 b$ ).

The parameters $2 \mathrm{~T}^{\prime} \|$ and $h_{0} / h_{-1}$ were plotted against
$\mathrm{pH}$. The maximum hyperfine splitting, $2 \mathrm{~T}^{\prime} \|$, decreases slightly from $55.7 \mathrm{G}$ at pH 7.4 to $54.4 \mathrm{G}$ at pH 5.0 for normal erythrocytes, and from $55.7 \mathrm{G}$ at $\mathrm{pH} 7.4$ to $54.9 \mathrm{G}$ at $\mathrm{pH} 5.0$ for DMD erythrocytes (data not shown). On the other hand, at $30^{\circ} \mathrm{C}$ the empirical parameter $h_{0} / h_{-1}$ changes abruptly at $\mathrm{pH}$ 5.9-5.6 in normal erythrocytes, although the change is gradual in DMD erythrocytes (Fig. 5). This difference in $\mathrm{pH}$ dependence of $h_{0} / h_{-1}$ between normal and DMD erythrocytes could not be explained by a difference in the amount of hemolysis, because essentially the same amount occurred in both erythrocytes within this range of $\mathrm{pH}$ (data not shown).

The temperature dependence of the empirical parameter $\mathrm{h}_{0} / \mathrm{h}_{-1}$ at $\mathrm{pH} 5.0$ in normal erythrocytes is in marked contrast to that at pH 7.4 (Fig. 6). Exposure of normal erythrocytes to $\mathrm{pH} 5.0$ causes much greater freedom of motion to the radicals and loss of both inflection points, always identified at $\mathrm{pH} 7.4$ in normal erythrocytes.

Reduction kinetics of the radicals of $\mathrm{I}(12.3)$ by ascorbate were studied. As can be seen in Fig. 7, the speed of destruction of radicals by ascorbate showed marked temperature dependence. The ESR signal of normal erythrocytes disappeared faster than that of DMD erythrocytes at temperatures studied here.

The statistical analysis of data obtained at $30^{\circ} \mathrm{C}$ shows that $15 \mathrm{~min}$ after addition of $5 \mathrm{mM}$ ascorbate, significantly higher amounts of spin label radicals remained in DMD erythrocytes than in normal erythrocytes. The spectra measured after approximately half of the ESR signal disappeared, were essentially the same as those of untreated erythrocytes (data not shown). Treatment of erythrocytes with phloretin resulted in elimination of the difference between normal and 

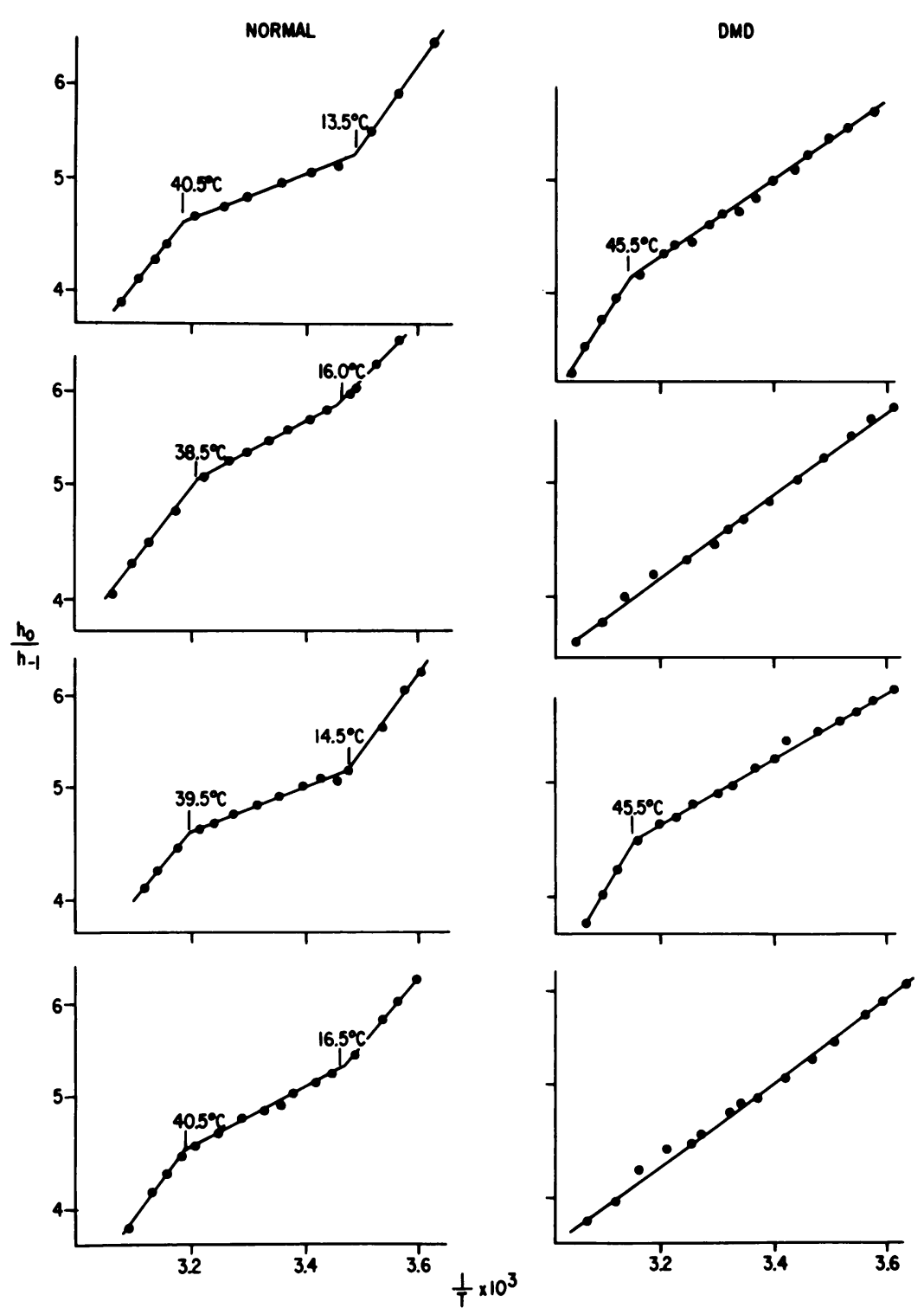

FIGURE 3 Effect of temperature of $h_{0} / h_{-1}$ of the spectra of $\mathrm{I}(1.14)$. The empircal parameter, $\mathrm{h}_{0} / \mathrm{h}_{-1}$, was calculated from the spectra of $\mathrm{I}(1.14)$ incorporated into normal and DMD patients' erythrocytes and plotted against the reciprocal of the absolute temperature. The figures on each chart indicate the temperature $\left({ }^{\circ} \mathrm{C}\right)$ of the inflection point. These data were obtained in four separate experiments. Each horizontal pair of curves represents a patient and his paired control.

DMD erythrocytes (Fig. 7). Treatment of I(12.3)-BSA complex with phloretin did not cause interference with the reaction between the radicals and ascorbate.

\section{DISCUSSION}

The spectra obtained by incorporating $\mathrm{I}(12.3)$ and I(5.10) into erythrocytes did not show a significant difference between samples obtained from normal subjects and those from DMD patients which confirmed the reported results $(16,17)$. On the other hand, the spectra of $\mathrm{I}(1.14)$ show quite a significant difference between the two types of erythrocytes. The spectra of $\mathrm{I}(1.14)$ presented here have been treated fundamentally as isotropic although there is some distortion by anisotrophy. When other biological membranes (mouse liver microsomes and mouse testis microsomes) are labeled with $\mathrm{I}(1.14)$, the spectra are very nearly those expected from isotropic motion. ${ }^{2}$ Jost

${ }^{2}$ Sato, B., K. Nishikida, R. A. Huseby, L. T. Samuels. Manuscript in preparation. 

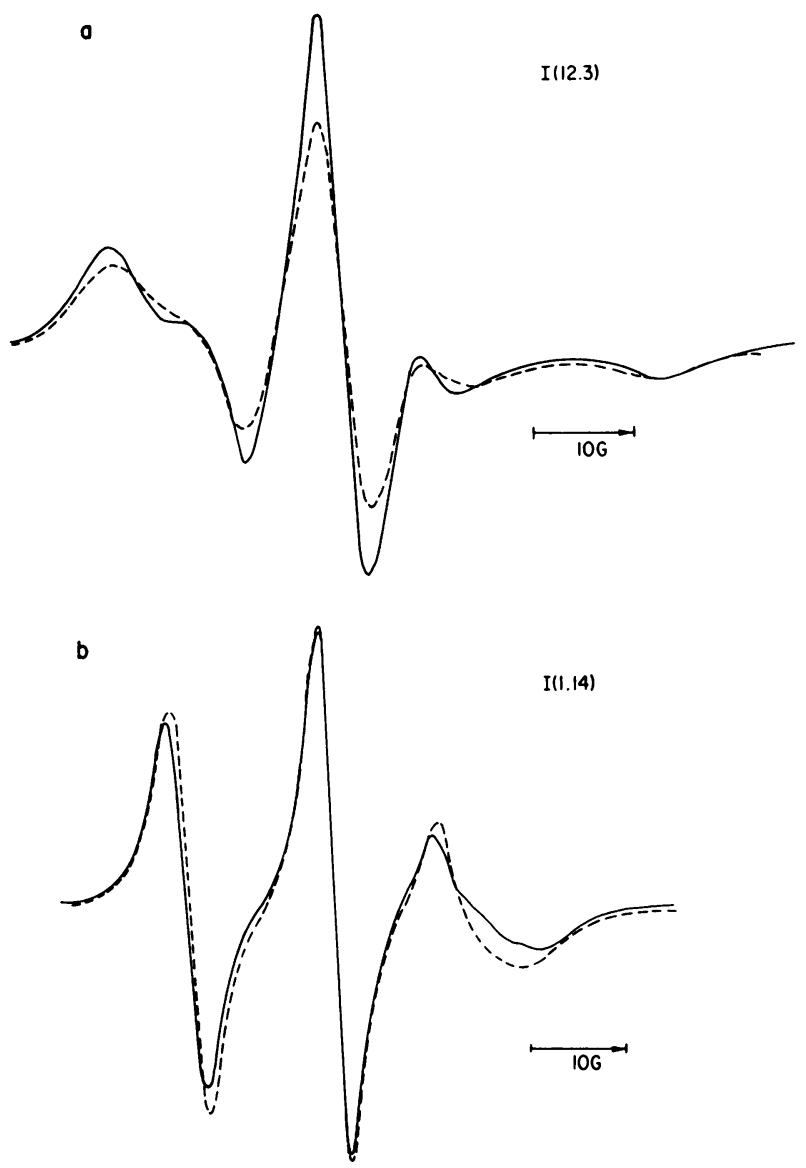

FIGURE 4 ESR spectra of I(12.3) of I(1.14) in normal erythrocytes at $\mathrm{pH} 7.4(-)$ and $\mathrm{pH} 5.0(---)$. The probes were incorporated and washed as described in Methods. An aliquot of these treated erythrocytes was washed twice more with 10 vol of $0.15 \mathrm{M} \mathrm{NaCl}-0.01 \mathrm{M}$ acetate, pH 5.0, or $10 \mathrm{vol}$ of Buffer I. The spectra were measured at $30^{\circ} \mathrm{C}$.

et al. (18) have demonstrated that tight interaction between $\mathrm{I}(1.14)$ and hydrophobic protein in a model membrane system gives the spectra an anisotropic pattern. Our results imply that isotropic spectra of I(1.14) incorporated into erythrocytes are distorted by the slow tumbling speed of the radicals, probably due to tight protein-lipid interaction. The tight protein-lipid interaction in the organization of the erythrocyte membrane was also suggested by using the ESR technique and perturbing the membrane proteins (19).

The empirical parameter, $h_{0} / h_{-1}$, used here for interpreting the spectra of $\mathrm{I}(1.14)$, clearly shows two inflection points on the temperature chart of normal erythrocytes. The precise cause of the inflections, however, is unclear. These results in normal erythrocytes correspond to the data observed by Bieri and Wallach (20). By analyzing paramagnetic quenching data, they suggested the appearance of new proteinphospholipid binding at $15^{\circ} \mathrm{C}$ and its disappearance

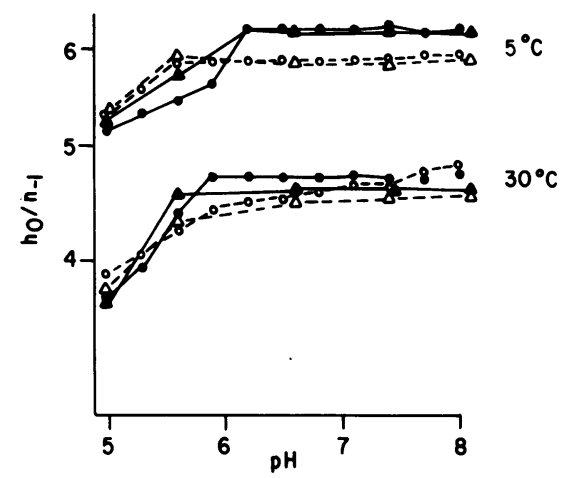

FIGURE 5 Effect of $\mathrm{pH}$ on $\mathrm{h}_{0} / \mathrm{h}_{-1}$ of the spectra of $\mathrm{I}(1.14)$. The

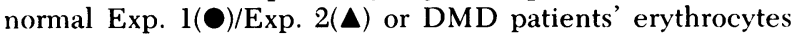
Exp. 1(O)/Exp. 2( $\triangle)$ were labeled with $\mathrm{I}(1.14)$ as mentioned in Methods. These treated erythrocytes were then equilibrated with $10 \mathrm{vol}$ of the various buffers adjusted to specific $\mathrm{pHs}$, centrifuged, and washed once with the buffer before recording spectra. The following buffer mixtures were adjusted to the different $\mathrm{pHs}$ within the ranges shown: $\mathrm{pH} 8.2-\mathrm{pH} 7.4,0.15 \mathrm{M}$ $\mathrm{NaCl}-0.01 \mathrm{M}$ Tris; $\mathrm{pH} 7.2-\mathrm{pH} 6.5,0.15 \mathrm{M} \mathrm{NaCl}-0.01 \mathrm{M}$ sodium phosphate; pH 6.2-pH 5.5, $0.15 \mathrm{M} \mathrm{NaCl}-0.01 \mathrm{M}$ maleate, acetate; $\mathrm{pH} 5.3-\mathrm{pH} 5.0,0.15 \mathrm{M} \mathrm{NaCl}-0.01 \mathrm{M}$ acetate.

above $40^{\circ} \mathrm{C}$. As reported here, the loss of inflection points by lowering $\mathrm{pH}$ to 5.0 , which might cause conformational change of proteins, also suggests the importance of the protein-phospholipid interaction for erythrocyte membrane organization.

The inflection point at $13.5^{\circ}-16.5^{\circ} \mathrm{C}$ seen in normal erythrocytes disappears in DMD erythrocytes. This result indicates that the domain in DMD erythrocyte membranes, where the radicals of $\mathrm{I}(1.14)$ are located, is quite different from that in normal erythrocytes. Generally, the vlaues of $h_{0} / h_{-1}$ in DMD erythrocytes are

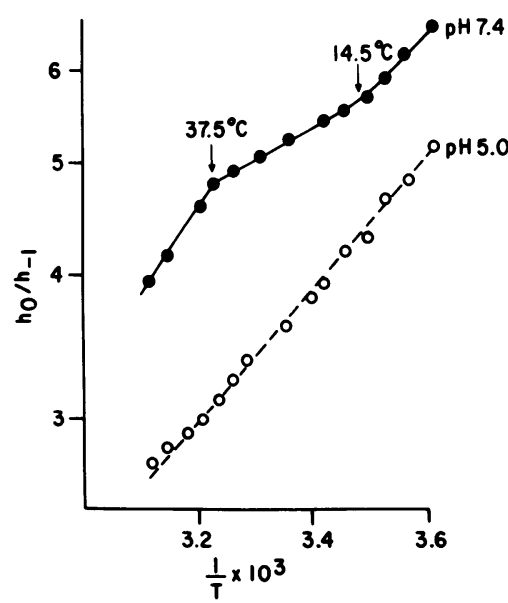

FIGURE 6 The difference in the temperature dependence of

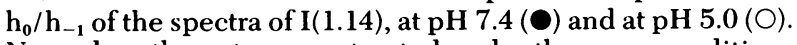
Normal erythrocytes were treated under the same conditions as described in Fig. 5. The spectra were recorded at the various temperatures. 


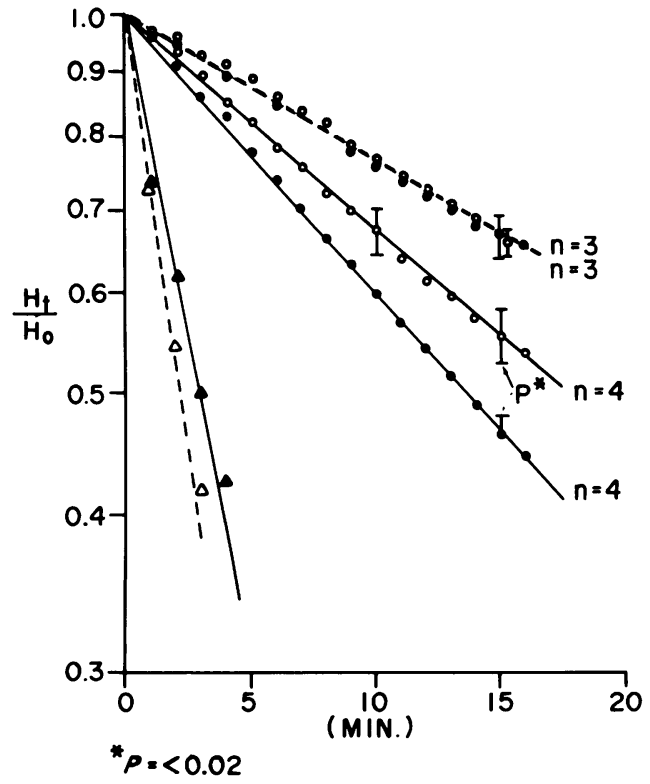

FIGURE 7 Effect of phloretin on reduction kinetics of the radicals of $\mathrm{I}(12.3)$ by ascorbate in normal or DMD patients' erythrocytes at $30^{\circ} \mathrm{C}$. The erythrocytes labeled with $\mathrm{I}(12.3)$ were divided into two parts. One part was preincubated with $0.5 \mathrm{mM}$ phloretin at room temperature $\left(22^{\circ} \mathrm{C}\right)$ for $90 \mathrm{~min}$, and another part was kept in Buffer I at room temperature. Both parts were then mixed with $5 \mathrm{mM}$ ascorbate, quickly followed by recording of the spectra at $30^{\circ} \mathrm{C}$. These data were obtained by using four different controls and four different patients in, four separate experiments, but in one experiment the erythrocytes were not treated with phloretin. normal erythrocytes, $\left(\mathrm{O}_{--} \mathrm{O}\right) \mathrm{DMD}$ erythrocytes, (- - normal erythrocytes treated with phloretin, $\left(0_{---}\right)$DMD erythrocytes treated with phloretin, (A) BSA-I (12.3) complex, $(\triangle)$ BSA-I(12.3) complex treated with phloretin. Values are given as means \pm SE. $n=$ number of individuals. Vertical lines represent $S E$ of the points indicated. $P$ value has been calculated by Student's small sample $t$ test.

lower than in normal erythrocytes, which also indicates that nonpolar domains in the DMD erythrocyte membrane are in a more fluid state than those in normal erythrocytes.

The membrane organization of intact erythrocytes has been reported to be relatively resistant to $\mathrm{pH}$ manipulation, using freeze-fracture electron microscopy (21). Exposure of intact erythrocytes to low pH did not result in aggregation of intramembranous particles, probably proteins extending into or through the membrane. However, treatment of isolated membranes from human erythrocytes at $\mathrm{pH} 5.5$ induced severe but reversible aggregation of the intramembranous particles (22). Lowering $\mathrm{pH}$ also has been demonstrated to induce a change of cell shape from discocyte to stomatocyte (23). These last observations imply that a change in membrane organization in both isolated membranes and intact erythrocytes could be induced by $\mathrm{pH}$ manipulation. Our results showing the $\mathrm{pH}$ dependence of the empirical parameter $h_{0} / h_{-1}$ of the spectra of $\mathrm{I}(1.14)$ incorporated into normal erythrocytes clearly demonstrate a change in membrane organization, although, as noted in Results, this $\mathrm{pH}$ treatment produced only a minimal change in the spectra of $\mathrm{I}(12.3)$.

The $\mathrm{pH}(5.5-5.9)$ which induces the abrupt change in $\mathrm{h}_{0} / \mathrm{h}_{-1}$ in normal erythrocytes at $30^{\circ} \mathrm{C}$ corresponds approximately to that which induces the agglutination of intramembranous particles in isolated membranes but not in intact membranes (22). The difference in $\mathrm{pH}$ effect between isolated membranes and intact erythrocytes, found in the electron microscope studies, might be related to the partial extraction of the erythrocyte membrane protein, spectrin (24). The data reported $(21$, 22) and those presented in this paper suggest that lowering $\mathrm{pH}$ results in loss of tight proteinphospholipid interaction in intact erythrocytes but that the particles remain uniformly distributed, possibly due to the effect of spectrin. The spectra of $\mathrm{I}(1.14)$ in intact erythrocytes at $\mathrm{pH} 5.0$, which did not show line-broadening, favor this view because redistribution and clustering of certain amounts of radicals cause the spin-spin interaction responsible for such broadening (25-27).

Turning to the $\mathrm{pH}$ dependence of the spectra of $\mathrm{I}(1.14)$ incorporated into DMD erythrocytes, the empirical parameter, $h_{0} / h_{-1}$, decreases gradually during the lowering of $\mathrm{pH}$ at $30^{\circ} \mathrm{C}$ compared to the abrupt change in normal erythrocytes. This seems to be further evidence of the difference in membrane organization between normal DMD erythrocytes.

The spectra of I(12.3) are essentially the same in both kinds of erythrocyte. We consider, however, that the spectra of I(12.3) in the erythrocytes were not suitable for making the difference in protein-phospholipid interaction clear, because $\mathrm{pH}$ reduction, which should induce structural changes in membrane proteins resulting in alterations of protein-phospholipid interaction, causes only a minimal change. The reduction kinetics of the radicals of $\mathrm{I}(12.3)$ in erythrocytes confirm this view. The radicals located in normal erythrocytes were destroyed significantly faster by ascorbate than those in DMD erythrocytes even though a significant difference in fluidity and coupling constant could not be demonstrated. There are two possible explanations of these results: $(a)$ the radicals locate in a deeper portion of the DMD membrane than in normal erythrocytes, or $(b)$ the diffusion rate of ascorbate into DMD membranes is slower than into normal membranes. We favor the second possibility for the following reasons: $(a)$ the carbonyl groups of $\mathrm{I}(12.3)$ which must be located in the outer membrane of the bilayer (28), should be anchored at the interface between polar and nonpolar regions, $(b)$ the measured coupling constant is the same in both 
erythrocytes, $(c)$ phloretin, which is known to interfere with permeability of hydrophilic nonelectrolytes into erythrocytes under the same conditions as those used here (29-31), causes marked delay of destruction of the radicals by ascorbate. Although exact mechanisms controlling the rate of ascorbic penetration into erythrocytes are unclear we consider that intact membrane organization in the erythrocyte facilitates ascorbate diffusion into the membrane.

The data presented here clearly show an abnormality of membrane organization in DMD erythrocytes. The observation of Matheson and Howland (2) showing changes in the shape of erythrocyte from discocyte to echinocyte was not confirmed by other groups (32). However, abnormal deformity of erythrocytes measured by elastimetry (5) and protein kinase alteration in the erythrocyte membrane (6) which has been successfully applied to the detection of DMD carriers (33), together with our results, confirm the current concept that $\mathrm{DMD}$ is basically a membrane disease involving many tissues.

\section{ACKNOWLEDGMENT}

This work was supported by U. S. Public Health Service research grants AM-00002 and CA-13291.

\section{REFERENCES}

1. Walton, J. N., and D. Gardner-Medwin. 1974. Progressive muscular dystrophy and the myotonic disorders. In Disorders of Voluntary Muscle. J. N. Walton, editor. Churchill (J. \& A.) Ltd., London. 561-613.

2. Matheson, D. W., and J. L. Howland. 1974. Erythrocyte deformation in human muscular dystrophy. Science (Wash. D. C.). 184: 165-166.

3. Probstfield, J. L., Y. Wang, and A. H. L. From. 1972. Cation transport in erythrocytes of Duchenne muscular dystrophy. Proc. Soc. Exp. Biol. Med. 141: 479-481.

4. Kunze, D., G. Reichmann, E. Egger, G. Leuschner, and H. Echhardt. 1973. Erythrozyten lipide bei progressiver muskeldystrophie. Clin. Chim. Acta. 43: 333-341.

5. Percy, A. K., and M. E. Miller. 1976. Reduced deformability of erythrocyte membrane from patients with Duchenne muscular dystrophy. Nature (Lond.). 258: 147-148.

6. Roses, A. D., M. H. Herbstreith, and S. H. Appel. 1975. Membrane protein kinase alteration in Duchenne muscular dystrophy. Nature (Lond.). 254: 350-351.

7. Butterfield, D. A., D. B. Chestnut, A. D. Roses, and S. H. Appel. 1974. Electron spin resonance studies of erythrocytes from patients with myotonic muscular dystrophy. Proc. Natl. Acad. Sci. U. S. A. 71: 909-913.

8. Butterfield, D. A., A. D. Roses, M. L. Copper, S. H. Appel, and D. B. Chestnut. 1974. A comparative electron spin resonance study of the erythrocyte membrane in myotonic muscular dystrophy. Biochemistry. 13: 5078-5082.

9. Butterfield, D. A., A. D. Roses, S. H. Appel, and D. B. Chestnut. 1976. Electron spin resonance studies of membrane proteins in erythrocytes in myotonic muscular dystrophy. Arch. Biochem. Biophys. 177: 226-234.

10. Hubbell, W. L., and H. M. McConnell. 1969. Motion of steroid spin labels in membranes. Proc. Natl. Acad. Sci. U.S.A. 63: 16-22.

11. Hubbell, W. L., and H. M. McConnell. 1969. Orientation and motion of amphiphilic spin labels in membranes. Proc. Natl. Acad. Sci. U. S. A. 64: 20-27.

12. Hubbell, W. L., and H. M. McConnell. 1971. Molecular motion in spin-labeled phospholipid and membranes. $J$. Am. Chem. Soc. 93: 314-326.

13. Wallach. D. F. H., and R. J. Winzler. 1974. Evolving strategies and tactics in membrane research. In SpinLabel Probes. D. F. H. Wallach and R. J. Winzler, editors. Springer-Verlag New York, Inc., New York. 304-343.

14. Stone, T. J., T. Buckman, P. L. Nordio, and H. M. McConnell. 1965. Spin-labeled biomolecules. Proc. Natl. Acad. Sci. U. S. A. 54: 1010-1017.

15. Ito, T., and S. Ohnishi. 1974. $\mathrm{Ca}^{++}$-induced lateral phase separation in phosphatidic acid-phosphatidylcholine membrane. Biochim. Biophys. Acta. 352: 29-37.

16. Butterfield, D. A., D. B. Chestnut, S. H. Appel, and A. D. Roses. 1976. Spin label study of erythrocyte membrane fluidity in myotonic and Duchenıe muscular dystrophy and congenital myotonia. Nature (Lond.). 263: 159-161.

17. Roses, A. D., S. H. Appel, D. A. Butterfield, S. E. Miller, and D. B. Chestnut. 1975. Specificity of biochemical and biophysical tests in Duchenne and Myotonic muscular dystrophy, carrier states, and congenital myotania. Trans. Am. Neurol. Assoc. 100: 131-134.

18. Jost, P. C., O. H. Griffith, R. A. Capaldi, and G. Vanderkool. 1973. Evidence for boundary lipid in membranes. Proc. Natl. Acad. Sci. U. S. A. 70: 480-484.

19. Wallach, D. F. M., S. P. Verma, E. Weidehamm, and V. G. Bieri. 1974. Hydrophobic binding sites in bovine serum albumin and erythrocyte ghost proteins. Study by spinlabeling, paramagnetic fluorescence quenching and chemical modification. Biochim. Biophys. Acta. 346: $68-81$.

20. Bieri, V. G., and D. F. H. Wallach. 1976. Lipid-protein relationships in erythrocyte membranes related by paramagnetic quenching of protein fluorescence. Biochim. Biophys. Acta. 443: 198-205.

21. Weinstein, R. 1974. The morphology of adult red cells. In The Red Blood Cell. D. M. Surgenor, editor. Academic Press, Inc. New York. 2nd edition. 213-268.

22. Pinto da Silva, P. 1972. Translational mobility of the membrane intercalated particles of human erythrocyte ghosts. J. Cell Biol. 53: 777-787.

23. Weed, R. I., and B. Chailley. 1973. Calcium-pH interactions in the production of shape change in erythrocytes. In Red Cell Shape. M. Bessis, R. I. Weed, and P. F. LeBlond, editors. Springer-Verlag New York, Inc., New York. 44-68.

24. Nicolsol, G. L., and R. G. Painter. 1973. Anionic sites of human erythrocyte membrane. II. Antispectrin-induced transmembrane aggregation of the binding site for positively charged calloidal particles. J. Cell Biol. 59: 395-506.

25. Sackmann, E., and H. Trauble. 1972. Studies of the crystalline-liquid crystalline phase transition of lipid model membranes. II. Analysis of electron spin resonance spectra of steroid labels incorporated into lipid membranes. J. Am. Chem. Soc. 94: 4492-4498.

26. Sackmann, E., H. Trauble, H. J. Galla, and P. Overath. 1973. Lateral diffusion, protein mobility, and phase transitions in Escherichia coli membranes. A spin label study. Biochemistry. 12: 5360-5369.

27. Ito, T., S. Ohnishi, M. Ishinaga, and M. Kito. 1975. Synthesis of a new phosphatidylserine spin-label and calcium-induced lateral phase separation in phospha- 
tidylserine-phosphadidyl-choline membranes. Biochemistry. 14: 3064-3069.

28. Kaplan, J., P. G. Cannonico, and W. J. Caspary. 1973. Electron spin resonance studies of spin-labeled mammalian cells by detection of surface-membrane signals. Proc. Natl. Acad. Sci. U. S. A. 70: 66-70.

29. Wolf, H. U. 1972. Studies on a $\mathrm{Ca}^{++}$dependent ATPase of human erythrocyte membranes. Effect of $\mathrm{Ca}^{++}$and $\mathrm{H}^{+}$. Biochim. Biophys. Acta. 266: 361-375.

30. Schatzmann, H. J., and G. L. Rossi. 1971. $\left(\mathrm{Ca}^{++}+\mathrm{Mg}^{++}\right)-$ activated membrane ATPase in human red cells and their possible relation to cation transport. Biochim. Biophys. Acta. 241: 379-392.

31. Triplett, R. B., J. M. Wingate, and K. L. Carraway. 1972. Calcium effects on erythrocyte membrane proteins. Biochem. Biophys. Res. Commun. 49: 1014-1020.

32. Miale, T. D., J. J. Frias, and D. L. Lawson. 1975. Erythrocytes in human muscular dystrophy. Science (Wash. D. C.). 187: 453.

33. Roses, A. D., M. J. Roses, S. E. Miller, K. L. Hull, and S. H. Appel. 1976. Carrier detection in Duchenne muscular dystrophy. N. Engl. J. Med. 294: 193-198. 